\title{
Oscillatory Regimes of Solutocapillary Marangoni Convection
}

\author{
Konstantin Kostarev ${ }^{1}$, Andrew Zuev ${ }^{1}$ and Antonio Viviani ${ }^{2}$ \\ ${ }^{1}$ Institute of Continuous Media Mechanics, \\ ${ }^{2}$ Seconda Università di Napoli \\ ${ }^{1}$ Russia, \\ 2Italy
}

\section{Introduction}

It is common knowledge that a fluid motion can be initiated both by the volume (buoyancy) forces, due to density variations in presence of a gravity field, and the surface (capillary) forces, due to variations of surface tension along a fluid/fluid interface. Involving in motion the surface and the near-surface layers the capillary forces gives rise to a volumetric flow generally known as the Marangoni convection. In turn, inhomogeneity of the surface tension may result from its dependence on temperature or concentration of the dissolved surfacetension active (surfactant) component. Such convection, thermocapillary or solutocapillary one, respectively, plays an important role in hydrodynamics and heat/mass transfer of inhomogeneous multi-phase media with the liquid/liquid interface or the free surface between liquid and gas. The Marangoni convection has a marked effect on the intensity of many technological processes encountered in food, chemical, oil, metallurgical and other industries, including those proceeding in microgravity conditions where the gravitational mechanisms of convective motion are weakened or absent. Particular interest on research in this field has quickened in recent years due to new achievements in the development of space technologies and life-support and survival systems for orbital stations. Marangoni phenomena is a problem of great application value for such areas as ecology (purification of water surface from petroleum products), meteorology, biology (motion of bacteria and microbodies), medicine (spreading of pulmonic surfactants during aerosol inhalation in treatment of lung diseases) and so on.

Despite the global abundance of the Marangoni convection the direct experimental investigation of the Marangoni flows in the so-called pure form is a challenging task. The reason is that under normal terrestrial conditions the existence of temperature and concentration gradients in a fluid also leads to development of the gravitational Rayleigh convection with considerably higher intensity than that of the Marangoni convection. Hence the latter proves to be almost suppressed by a more severe convective driving mechanism or veiled by its action. At the same time, under certain conditions, the surface forces may dominate over the buoyancy ones. Such conditions are realized, for example, in shallow layers and fluid films, liquid bridges and zones confined between solid surfaces, and also for small insoluble drops or gas bubbles suspended in liquids. In the past decades, the Marangoni convection in such systems has been the subject of intensive investigation. However, most of the studies are concerned with thermocapillary variant of the Marangoni 
convection whereas the works on solutocapillary convection are practically absent. The reason is that solutocapillary flows are more complicated than thermocapillary ones and are dependent on a variety of factors impeding their theoretical and numerical analysis as well as experimental examination. Indeed, experimentally the concentration gradients are more difficult to generate and sustain than the temperature gradients. The absence of simple and adequate methods for measuring local surfactant concentration at the free surface also presents serious problems. The other challenge is a non-stationary nature of solutal flows associated with the diffusion processes. The action of some additional side effects related with dissolution of the surfactant in a liquid, its evaporation and transition into a gas phase and adsorption at the interface also add complexity to treatment of this problem.

Despite an obvious similarity of the driving mechanisms, the thermocapillary and solutocapillary Marangoni phenomena were found to be not completely analogous. The key difference is that the characteristic times of the admixture diffusion are hundred times longer than times of heat diffusion and so the values of Marangoni number, characterizing the intensity of solutocapillary convection, increase many times. This changes drastically the ratio of contributions to mass transfer made by competing gravitational and Marangoni mechanisms. As a result, the concentration inhomogeneities in the fluid exist for much longer time, whereas the intensity and duration of the capillary forces at the interface show a manifold increase. The other marked distinction of the solutocapillary convection consists of surfactant adsorption which caused an increase in the local concentration of the surfactant and its redistribution over the surface, equalizing surface tension inhomogeneity. These differences give rise to new phenomena that cannot occur in thermocapillary situation.

Thus, the results of experimental observations show that if an upward-directed temperature gradient is maintained in a liquid, a thermocapillary flow forms around a bubble in the form of an axisymmetric toroidal vortex with a vertical symmetry axis. Under the action of Marangoni forces, the liquid flows along the bubble-free surface to its lower pole and slowly rises at some distance, creating a returning flow. Correspondingly, in any vertical plane drawn through a diameter, two stationary vortices that are symmetric with respect to the bubble are observed. The vertically stratified temperature distribution in the liquid also remains stationary. In contrast to thermal Marangoni convection, the described below our experiments (Birikh et al., 2006, 2008; Bushueva et al., 2008; Kostarev et al., 2004, 2006, 2007, 2008a, 2008b, 2009a, 2009b; Zuev et al., 2006, 2008a, 2008b) revealed the self-oscillatory regimes of solutal convection in an aqueous solution of surfactant with vertically stratified concentration, and the threshold character of excitation of solutocapillary flows near the free bubble surface.

\section{Experimental approach and apparatus}

Visualization of non-homogeneous distribution of the surfactant concentration in the fluids mixture was made by means of interferometer observations. Investigations were made in a thin liquid layer, filling the gap between two plane-parallel glasses. On the side of the fluid the glasses were covered by a semi-transparent mirror coating to provide repeated passage of the object beam through the fluid in the cavity. Thus, the glass plates served as the walls of the shallow $90 \times 40 \mathrm{~mm}$ rectangular cavity (the so-called Hele-Shaw cell) forming the working cell of the Fizeau interferometer, which was adjusted to a single infinitely wide fringe. It is to be noted that this particular structure of the cuvette intended for a simultaneous formation of the reference and a series of the object light beams was first used 
in (Gustafson et al., 1968, Marsters \& Advani, 1973) for studying the mass transfer processes. In such a cuvette, the interference pattern is formed only due to inhomogeneity of the medium enclosed between the mirror coatings of the cuvette walls and is not affected by thermal deformations of the interferometer elements. Therefore the obtained picture reflects the real state of the fluid.

The interferometer allowed visualizing anomalies in the solution concentration as a pattern of isolines of the refraction index, which under isothermal conditions varied proportionally to the concentration. For a liquid layer $2 \mathrm{~mm}$ thick, the difference in the values of the refraction index between two adjacent monotonous interference fringes was $0.27 \times 10^{-3}$. With account of the non-linear dependence of the refraction index on concentration (Vazquez et al., 1995, 1997) this corresponds to a $0.3-0.7 \%$ variation in the solution concentration, depending on the kind of used surfactant. The maximum error of the concentration measurements did not exceed $0.1 \%$. As the thickness of the cavity gap did not exceed $2 \mathrm{~mm}$, which is 10 times smaller than other cavity sizes, the optical inhomogeneity along the propagation path of the object beam are neglected and the structure of the concentration fields and the flow streams is considered to be two-dimensional. The scheme of the experimental setup is shown in Fig. 1. The observations can be made both in reflected and transmitted light. The experimental cell can be oriented either horizontally (position I) or vertically (position II). In tests with horizontally oriented cells the collimator was placed in font of the mirror inclined at an angle of $45^{\circ}$. All experiments were carried out at the ambient temperature $(20 \pm 1)^{\circ} \mathrm{C}$.

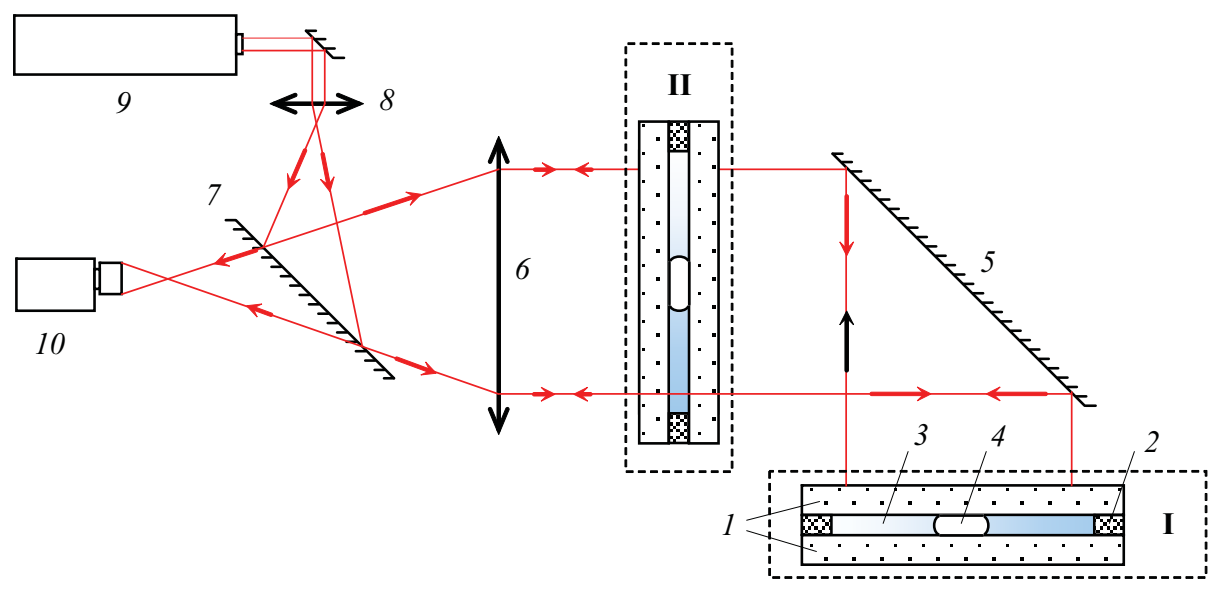

Fig. 1. Experimental setup: 1 - semi-transparent mirror-plated glasses; 2 - sectionalizing plates; 3 - fluid layer; 4 - gas bubble or insoluble droplet; 5 - mirror, 6 - objective; 7 - semitransparent mirror; 8 - diaphragm; 9 - laser; 10 - camera-recorder

\section{Two horizontal layers of soluble fluids}

In our first experiment (Kostarev et al., 2004) was studied a two-layer system of mutually miscible fluids with different surface tension. For this effect we used water and aqueous solutions of some liquid surfactants such as acetic acid with mass concentration ranging from 0 to $70 \%$, or methyl, isopropyl, and ethyl alcohols with $50-100 \%$ concentration. The 
experimental cells of thickness $\geq 2 \mathrm{~mm}$ was first set in a vertical position and filled sequentially by two solutions of various concentration. Due to the small values of the fluids diffusion coefficient these solutions did not mix, only the very narrow diffusion zone between the liquid layers was formed in the cell. After this the cell was placed horizontally. Horizontal positioning of the cell caused a lighter fluid to flow over a heavier one. As a result, we obtained a liquid medium with a vertical surfactant concentration difference. Note that density of the acetic acid is higher and density of the alcohols is lower than that of water. This allowed us to realize situations when a surfactant layer was formed either above or under the water layer, or differently, the situation leading to formation of downwarddirected or upward- directed surface tension gradient at the bubble surface.

Before the bubble was injected into the fluid the interference pattern displayed a monochrome image field because in the cell set in a horizontal position the direction of the transmitted optical radiation coincide with the direction of the density gradient of nondisturbed fluid system. Air bubbles, injected into a fluids system by a medical syringe, took the form of a flat disk 5-15 mm in diameter, squeezed between the horizontal cell walls. Due to the vertical concentration difference the solutocapillary Marangoni flow must form along the free lateral surface of the bubble. Under these conditions the surfactant was transported by the solutocapillary forces to the lower (in experiments with the methyl alcohol) or upper (in experiments with acetic acid) areas of the bubble boundary. The transferred lighter or denser liquid fraction has to partly dissolve in the ambient liquid and partly return (ascend or sink, respectively), creating a circulating convective current. However, unlike a thermal convection, which basically had the form of a stationary axially symmetric vertical vortex, the initiated solutal convection was of well-pronounced oscillatory nature.

The evolution of this convection mode is illustrated by a series of interferograms in Fig. 2. At first, after insertion of the air bubble no motion is observed in the surrounding liquid. The concentration field around the bubble does not change either and looks like a uniform field (Fig. 2,a). Some time later the bubble turns out to be surrounded by a system of the concentric concentration isolines (Fig. 2,b) caused by an abrupt ejection of the excess surfactant accumulated under the action of solutocapillary forces at the lower or upper surface of the bubble. Then, as the buoyancy force restores the vertical density stratification of the solution this disturbance of the concentration field gradually disappears (Fig. 2,c) and the interference pattern again changes into a monochrome picture.

In our experiments this process was repeated again and again with a fairly good periodicity and continued until all liquid around the bubble was mixed to a homogeneous medium. The period $T$ of these oscillations (defined as a time interval between two outbursts of the intensive convection) ranged from several seconds to minutes and depended on time, layer thickness, horizontal diameter of the air bubble, the initial concentration gradient and physical properties of the liquids. Fig. 3 shows the variation of the oscillation frequency with time in a two-layer system composed of water and $40 \%$ aqueous solution of acetic acid for bubbles with diameters from 2.4 to $15.0 \mathrm{~mm}$. It is seen that as the solution is mixed and the vertical concentration gradient decreases, the period of oscillations initially equaling $10 \mathrm{sec}$ gradually increases (frequency, respectively, decreases), and the oscillations occur more and more seldom, until after approximately $10 \mathrm{~min}$ they come to an end. However, the graph does not show any essential effect of the bubble diameter on the oscillation frequency, which only slightly decreases with a growth of the bubble diameter. Moreover, oscillations of smaller bubbles stopped in a shorter time. 
a)

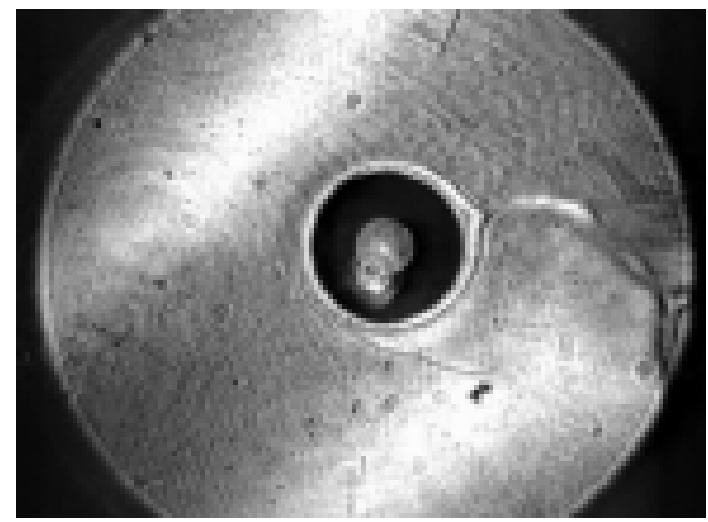

b)

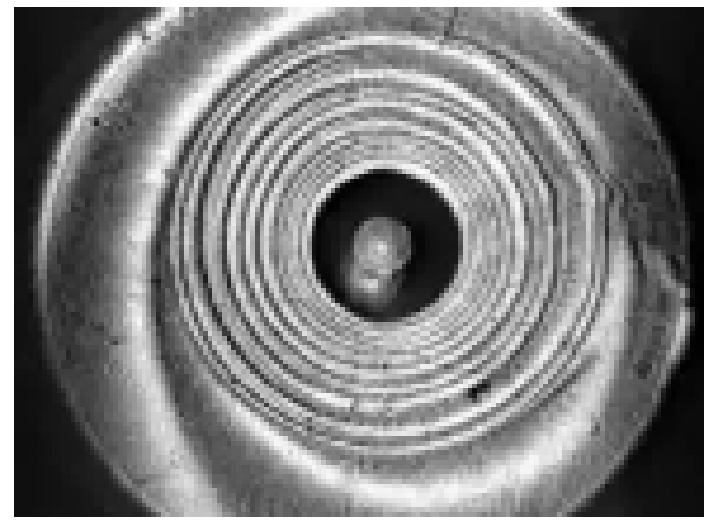

c)

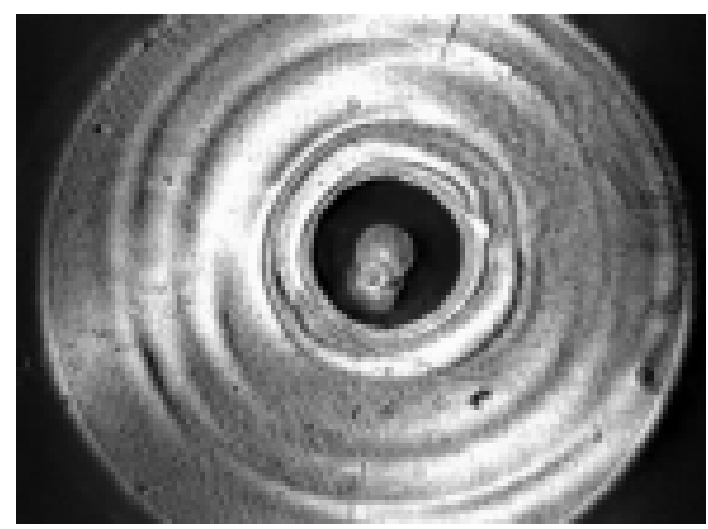

Fig. 2. Evolution of the concentration field disturbance around the air bubble in a horizontal layer of the aqueous isopropanol solution. Layer thickness $h=2.6 \mathrm{~mm}$, concentration difference $\Delta C=20 \%$, bubble diameter $d=5.0 \mathrm{~mm}$. $t$, sec: $0(a), 2.0(b), 10.0(c)$. View from above 


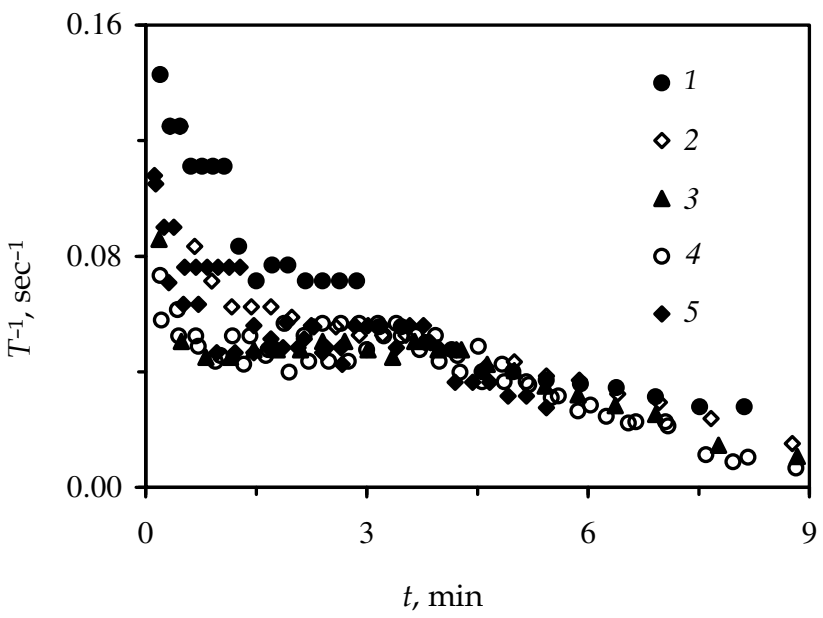

Fig. 3. Time variation of the oscillation frequency in two-layer system composed of water and $40 \%$ acetic acid solution for bubbles of various diameters $d, \mathrm{~mm}: 15.0$ (1), 10.7 (2), 7.1 (3), $5.1(4), 2.4(5)$

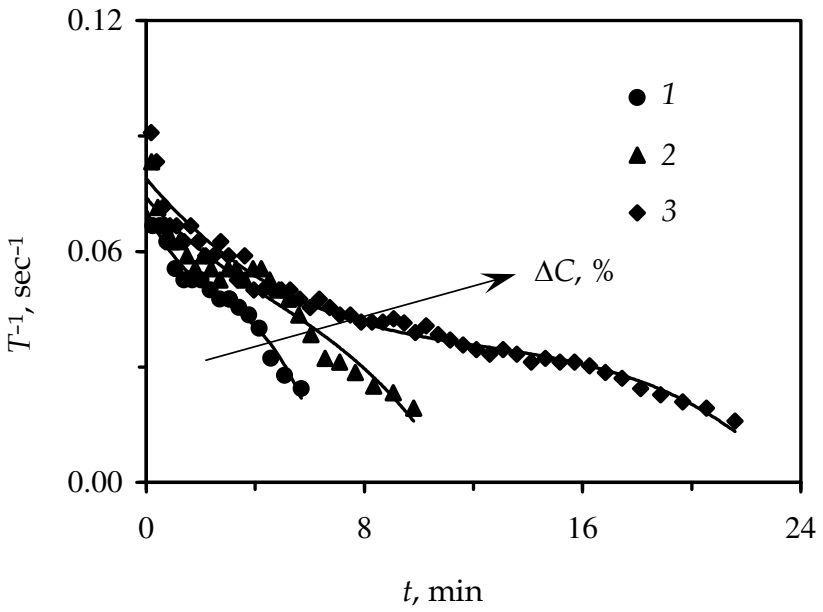

Fig. 4. Time variation of the oscillation frequency in two-layer system composed of water and $40 \%$ acetic acid solution. Bubble diameter $d=4.8-5.9 \mathrm{~mm}$. Initial concentration difference $\Delta C, \%: 20$ (1), 40 (2), 70 (3)

Evidently the oscillation frequency is chiefly defined by the layer thickness (i.e., the vertical bubble size) and the concentration gradient. With a decrease of the initial concentration difference (Fig. 4) the oscillation period slightly increases. At the same time, duration (the lifetime) of the oscillations significantly increases with a growth of the initial concentration difference. The specific feature of the observed oscillations is that they decay quite unexpectedly despite the fact that the fluid still preserves some vertical concentration 
gradient. To a larger extent the discovered phenomenon was found to depend on the choice of the surfactant and its concentrations. Thus, more pronounced oscillations with fairly large periods (hundreds of seconds and more) were observed in the experiments with water and $15-60 \%$ solutions of acetic acid. At lower surfactant concentrations the oscillations did not occur at all, whereas at essentially higher concentrations (more than 60\%) the oscillations were so frequent that they merged into one continuous oscillation mode in the form of "boiling" of the surfactant-saturated solution around the bubble. On the contrary, in tests with alcohol solutions, the concentration range giving rise to essential oscillations was much narrower and "boiling" was the main mechanism of mass transfer. Evidently, the period and lifetime of the oscillations are defined not by the concentration difference but by the difference in the values of the surface tension, which in most cases depends nonlinearly on the solution concentration.

The experimental findings suggest that solutocapillary flows are initiated in a threshold manner. To ensure their further development it is essential that some parameters specified both by the problem geometry and by physical properties of the examined liquids (mainly surface tension, density and viscosity) should reach critical values. However, such an experimental scheme - a combination of the vertical direction of observations (top view) and the horizontal orientation of the liquid layer - did not allow us to investigate directly the structure of convective flows and the evolution of the vertical distribution of surfactant concentration in the fluid. Therefore, our next study was focused on solutocapillary motion developed around the air bubbles and insoluble drops placed into a thin vertically situated liquid layer filled by aqueous surfactant solution with uniform vertical gradient of concentration.

\section{Vertical layer with a stratified solution}

The experiments were performed in order to visualize the vertical structure of convective flows and concentration fields (Zuev et al., 2006). The experimental cell was set vertically on its narrow face and filled in turn half by water and half by surfactant solution. The aqueous solutions of isopropyl alcohol with the concentration range from 10 to $50 \%$ or of acetic acid with the concentration range from 30 to $70 \%$ were used. After some time the diffusion between the fluids led to the formation of an area with a stable vertical stratification of surfactant concentration. The gas bubble or a drop of insoluble fluid was placed in a center of a thin (1.2 mm thick) gap between vertical cell walls with the help of medical syringe. As the initial bubble/drop diameter was larger than the layer thickness, they were squeezed between the cell walls so that took the form of a short cylinders with a free lateral surface. A special wire frame in the form of a flattened thin ring was used to prevent the bubble from buoying up under the action of the Archimedean force and to hold it in a fixed state in the cell center. The frame left the major part of bubble/drop lateral surface free and did not hamper the development of the Marangoni convection. Observations were made on the side of wide cell faces. Due to the very small cell thickness the arising flows and concentration distributions around the bubble/drop interface can be considered to be two-dimensional.

In the absence of the bubble the interference pattern of the concentration field in the stratified solution was presented by a system of horizontal lines. The injection of the bubble essentially disturbed the concentration distribution. Formation of the concentration gradient resulted in the appearance of tangential solutocapillary forces at the bubble lateral surface, which caused the development of the Marangoni convection. Fig. 5 presents the typical 
interference pattern of the concentration field around the bubble in the heterogeneous fluid mixture, formed at the top by water and at the bottom by the $70 \%$ acetic acid solution (downward surfactant concentration gradient). The direction of the surface tension gradient is opposite to the direction of surfactant concentration gradient which implies the origin of solutocapillary capillary forces directed to the upper pole of the bubble. The height of the bubble, which was slightly deformed by the gravitational force, was $3.8 \mathrm{~mm}$, and its width $6.0 \mathrm{~mm}$. At first, the convective flow near the bubble was quite similar to that observed in thermocapillary experiment (Kostarev et al., 2006). At a distance from the bubble the interference pattern of the concentration field in the solution was presented by a system of horizontal lines. At the bubble surface under the action of tangential solutocapillary forces the solution saturated by the surfactant moves from the lower pole region upwards forming a thin layer round the bubble (Fig. 5,a). In contrast to a thermocapillary convection, due to long characteristic times of diffusion the excess of the surfactant transferred along the bubble surface could not fully dissolve in the surrounding fluid. As a result the solution with high surfactant concentration was accumulated near the upper pole in the form of a peculiar "cap", whereas the layer with homogeneous concentration formed around the bubble eliminated the concentration difference between the poles - the source of the Marangoni convection. As soon as the ascending solutocapillary motion along the bubble surface vanished, the dynamic equilibrium of the concentration "cap", which was heavier than the surrounding fluid, was disturbed. Under the action of the Archimedean forces the streams of the acetic acid began to move downward on the right and on the left of the bubble. Due to fluid continuity this motion, in tern, was accompanied by formation of an upward-directed stream under the bubble carrying the solution with a higher surfactant concentration to the lower pole of the bubble (Fig. 5,b).

The recovery of the surfactant concentration difference between the bubble poles triggered again the Marangoni forces which abruptly increased the rising flow. As a result an intensive convection motion was initiated near the lateral surface of the cylindrical bubble in the form of two symmetric vortices. During evolution these vortices captured and mixed the larger volume of the solution with high surfactant concentration. As a result the average density of the solution within the vortices increased, they shifted downwards and at a certain moment cut off the surfactant supply from the bubble lower pole (Fig. 5,c). Once this happens, the convection motion ceased as fast as it had started. The whole process of evolution and decay of the intensive motion around the bubble lasted not longer than $30 \mathrm{sec}$. Later under the action of the gravity forces the vertical concentration stratification gradually recovered approaching the initial state. Then, after a time, the whole process was repeated. Such oscillations of the fluid motion occurred with the period of about 1-2 min and could continue as long as the external stratification of the surfactant concentration remained intact - about several hours. Gradually an area of the homogeneous solution formed under the bubble due to a mixing action of the vortex cells and extended in length with time. However as vertical concentration gradient near the bubble still exists, the oscillations are periodically reinitiated, though with less intensity.

In experiments with isopropyl alcohol, in which the gradient of the surface tension had an opposite direction (water is below), the arising structure of the concentration field and flows was bilateral symmetric: the surfactant on the bubble surface was carried to its lower pole and periodically initiated convective vortices rotated in the opposite direction (Fig. 6). In this case, a homogeneous zone was formed over the bubble. The surfactant concentration gradient near the bubble gradually decreased, so did the intensity of the arising vortices. 
a)

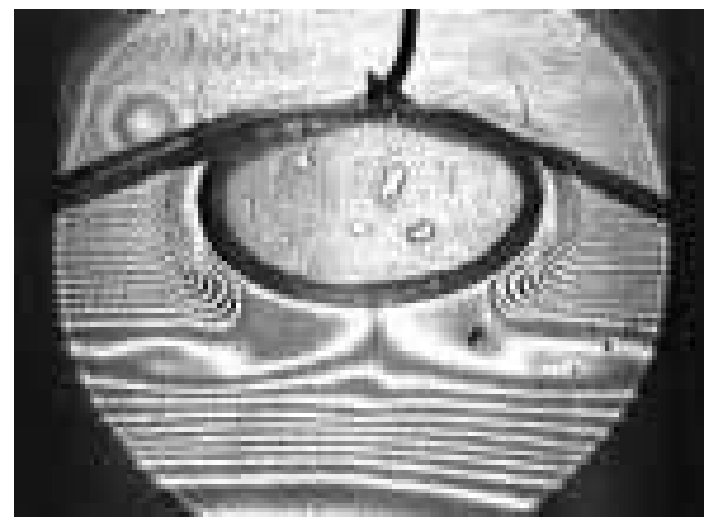

b)
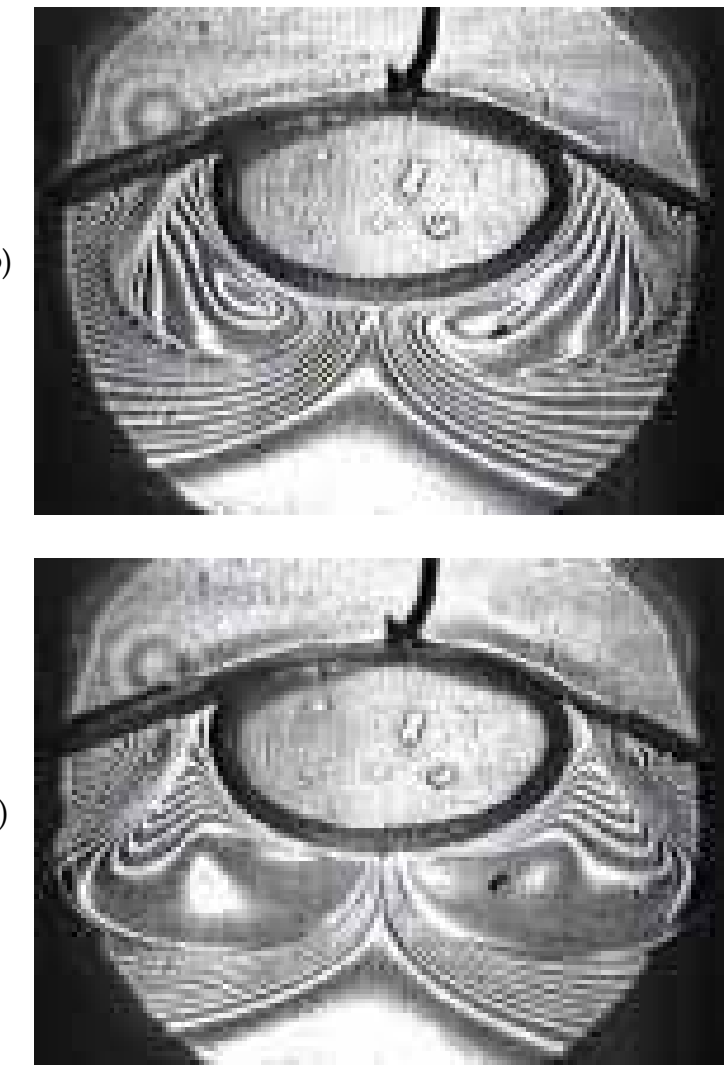

Fig. 5. Interferograms of concentration distribution around the air bubble in vertical layer of stratified acetic acid solution. Concentration difference between the bubble poles $\Delta C=10 \%$, vertical diameter of the bubble $d=3.8 \mathrm{~mm}$. Time elapsed from the beginning of a cycle $t$, sec: $0(a), 4.50(b), 8.25(c)$. View from the side 
a)

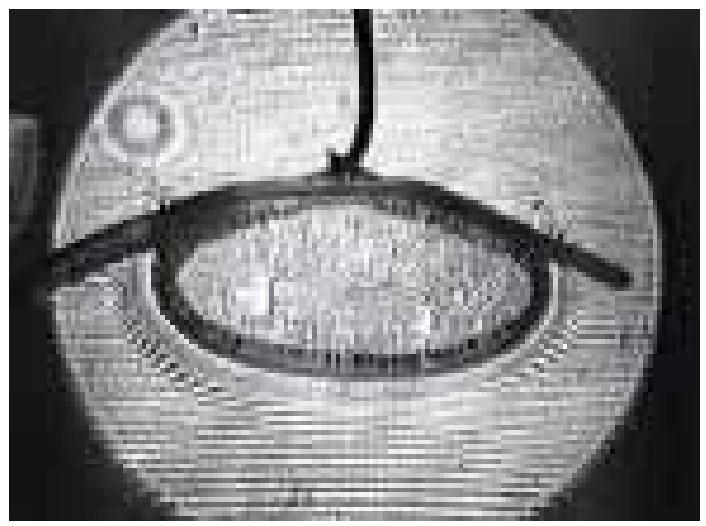

b)

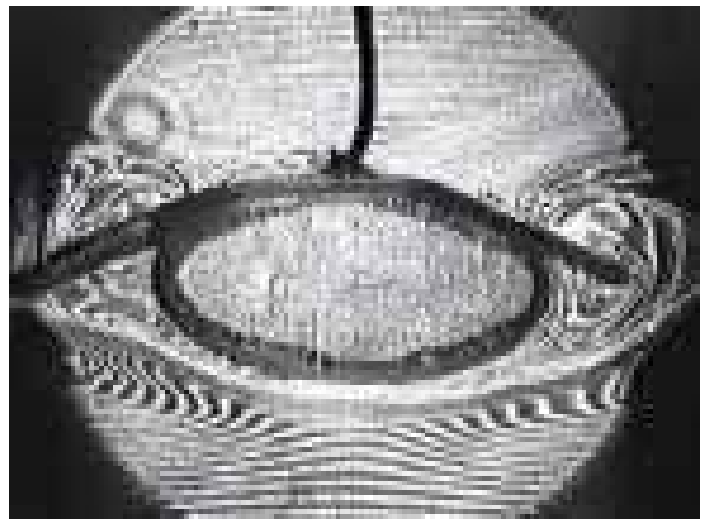

c)

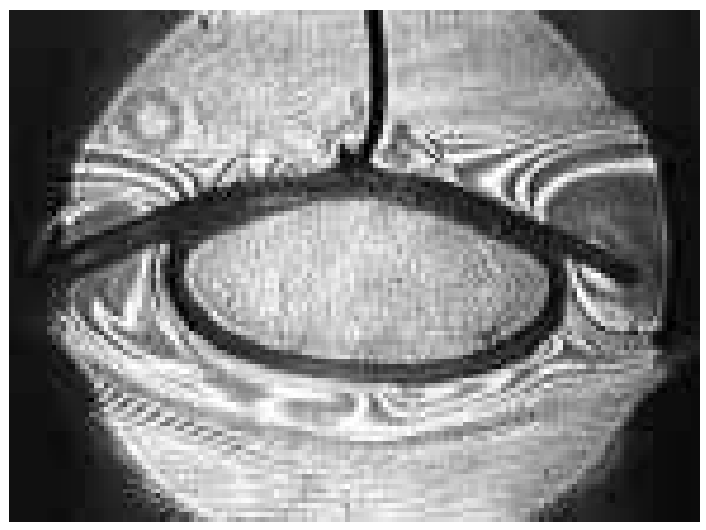

Fig. 6. Interferograms of concentration field around the air bubble in vertical layer of stratified isopropyl alcohol solution. Concentration difference between the bubble poles $\Delta C=4 \%$, vertical diameter of the bubble $d=3.8 \mathrm{~mm}$. Time elapsed from the beginning of a cycle $t$, sec: $0(a), 2.0(b), 10.0(c)$. View from the side 
The videotape recording of interferograms allowed us to trace the evolution of concentration field around the bubble. The measurements of the concentration distribution on the vertical coordinate were made at the time moments preceding the beginning of each cycle of oscillations when the intensity of the convective motion was minimal. In the system "water - 70\% acetic acid solution" concentration decreases monotonically with liquid layer height. Then the bubble began to stir actively the surrounding fluid, reducing the concentration difference between the bubble poles and the mean concentration of the solution under the bubble. At the initial time the vertical concentration gradient $\nabla C$ was a maximum and equal to $6.5 \% / \mathrm{mm}$. The corresponding values of the diffusion Marangoni $M a$ and Grashof $\mathrm{Gr}$ numbers were $4.1 \times 10^{6}$ and $1.9 \times 10^{3}$. Then the surfactant concentration gradient diminished rapidly with time, reaching the minimum value $4.4 \% / \mathrm{mm}$ $\left(M a=3.0 \times 10^{6}, G r=1.4 \times 10^{3}\right)$ approximately $60 \mathrm{~min}$ after the beginning of oscillations. After this it again increased approaching some constant asymptotic value of $5.2 \% / \mathrm{mm}$ $\left(\mathrm{Ma}=3.1 \times 10^{6}, \mathrm{Gr}=2.0 \times 10^{3}\right)$.

Analogous studying of the evolution of the concentration fields and the concentration gradient averaged over the bubble height was made for the "water- $40 \%$ isopropyl alcohol solution" system. In this case in contrast to the solutions of acetic acid a decrease and the following increase of the concentration gradient manifest themselves to a lesser degree. Such a behavior of the gradient is evidently due to the fact that with decrease in the intensity of solutocapillary motion the recover of vertical concentration gradient near the bubble due to gravitation and diffusion occurs much slower in alcohol solutions owing to a greater viscosity and a smaller diffusion coefficient. In experiments with lesser concentrated $20 \%$ alcohol solutions such extremum is not observed any longer, and the gradient is merely decreasing in a monotonic manner from $1.0 \% / \mathrm{mm}\left(\mathrm{M} a=16.8 \times 10^{6}, \mathrm{Gr}=1.8 \times 10^{3}\right)$ to $0.3 \% / \mathrm{mm}\left(\mathrm{Ma}=3.5 \times 10^{6}, \mathrm{Gr}=0.4 \times 10^{3}\right)$.

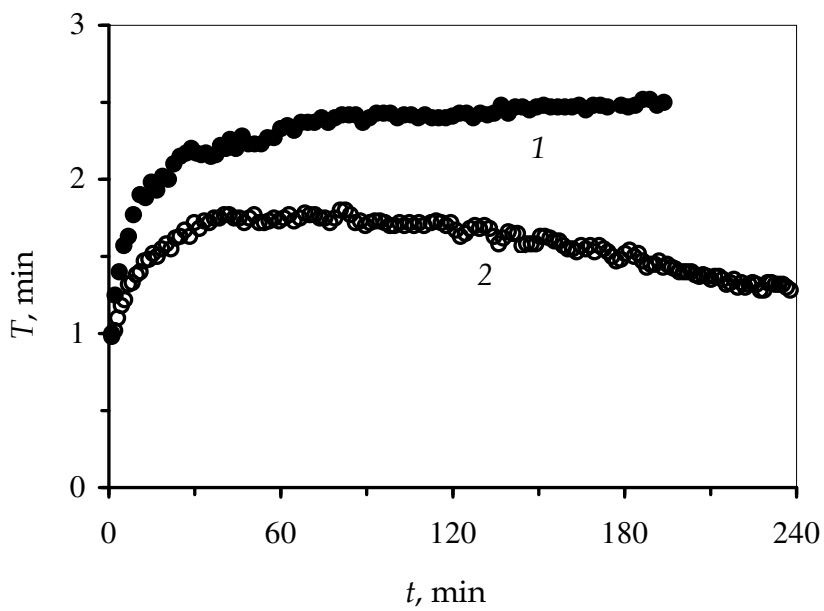

Fig. 7. Time dependence of the oscillation period. 1 - isopropyl alcohol solution, 2 - acetic acid solution

The time dependence of the oscillation period was investigated for different values of the average solution concentration, the concentration gradient and the diffusion Marangoni and 
Grashof numbers. It has been found that the period of convective oscillation changed simultaneously with a variation of the concentration gradient. In tests with $20 \%$ isopropyl alcohol solution the period increased monotonically with time and reaches the maximum within the first hour, after which it remained unchanged (curve 1 in Fig. 7). In tests with acetic acid solution the oscillation period within the first hour also increased (Fig. 7, curve 2), but then began to decrease, which implies that the time dependence of the period was the inverse of that of the concentration gradient. Thus in both cases there existed proportionality between the frequency (inverse period) of oscillations and concentration gradient of the surrounding fluid. In Fig. 8 the dimensionless frequency, normalized to the concentration Marangoni number, is plotted on the y-coordinate and the dimensionless time is plotted on the $\mathrm{x}$-coordinate. As evident from the graph as soon as the oscillation are set up the ratio $(T / \tau)^{-1} \cdot M a^{-1}$ turns out to be of the same value for different fluids and remains constant during the whole experiment not depending on the orientation of the concentration gradient (all experimental points for both states are well located on the straight line, which is nearly parallel to the time axis). The obtained result supports the view that just the solutocapillary Marangoni forces are the main initiator of periodical convective motion around the bubble in the heterogeneous solution of fluid surfactant.

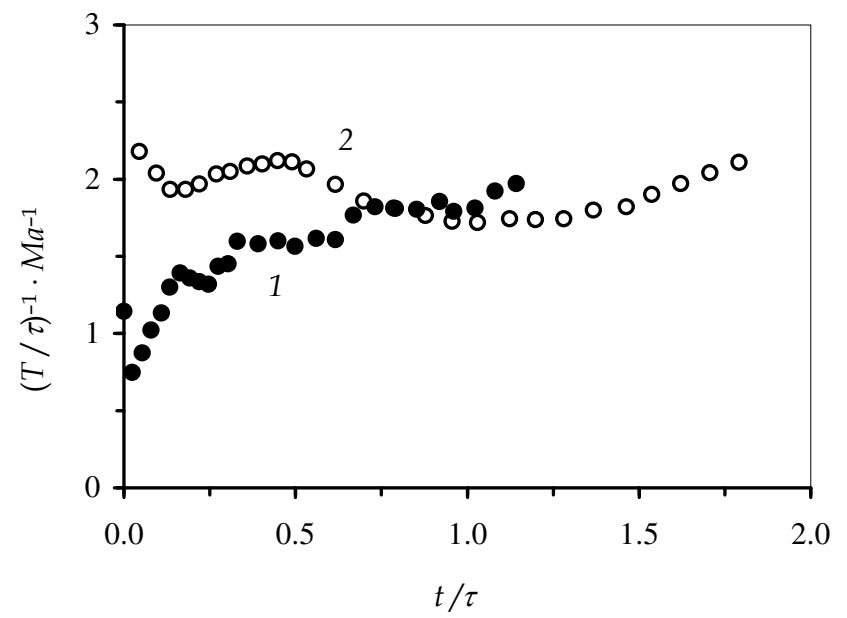

Fig. 8. Dimensionless oscillation frequency. 1 - isopropyl alcohol solution, 2 - acetic acid solution

The described oscillations of the solutal flow were also observed near the interface between two mutually insoluble fluids (Kostarev et al., 2007). For experimental investigation of this case we used the chlorobenzene drop placed in an aqueous solution of isopropanol. Note that isopropanol being surfactant for water and chlorobenzene is readily dissolved in both fluids, whereas the top solubility of pure chlorobenzene in water is $0.05 \%$ (at $30^{\circ} \mathrm{C}$ ) only and that of water in chlorobenzene is even less. It means that in the process of mass transfer the drop should absorb only one of the mixture components - a surfactant. Fig. 9 shows a series of interferograms, describing evolution of isopropyl alcohol distribution inside and around the drop of chlorobenzene in water during one of the cycles of the oscillatory mode. The concentration gradient in the stratified isopropyl solution was directed upward. The 
isopropanol, being soluble in both fluids, diffused easily through the interface in both directions.

a)

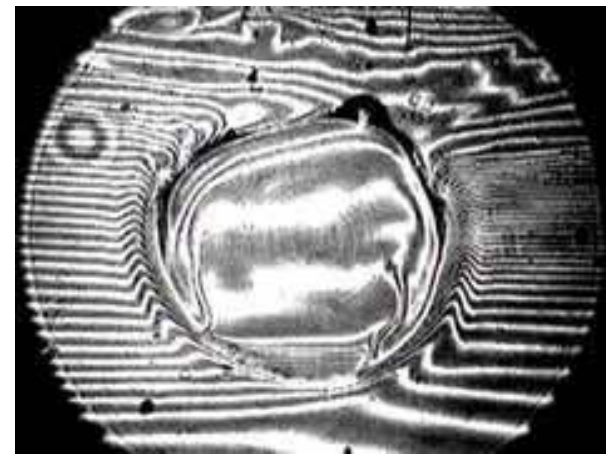

b)

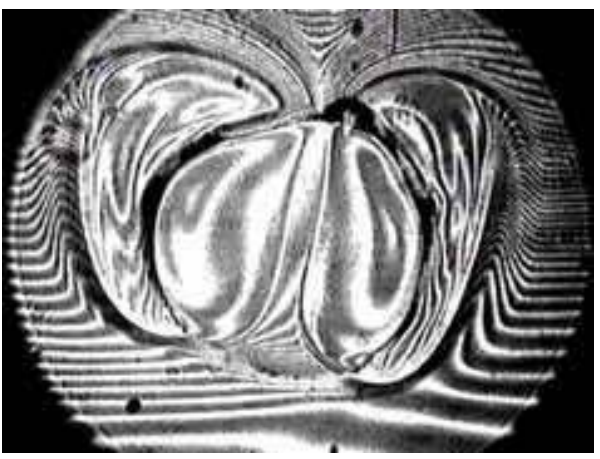

c)

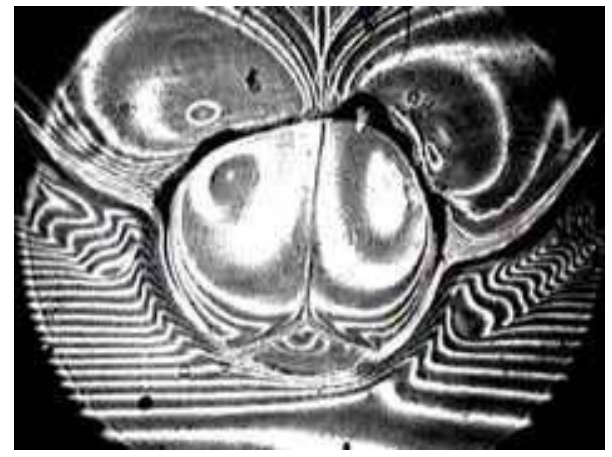

Fig. 9. Interferograms of the concentration field inside and around the drop of chlorobenzene absorbing isopropyl alcohol from its aqueous solution. Difference of alcohol concentration between the drop poles $\Delta C=8 \%$, drop diameter $d=6.0 \mathrm{~mm}$. Time elapsed from the beginning of the cycle $t$, sec: $0(a), 3(b), 18(c)$

The ease, with which the surfactant penetrates the surface, imparts some peculiar features to the development of motion in the system of fluids. Thus, a greater part of the drop surface is constantly in slow motion, because, due to absorption of the alcohol by the drop, there is always a gradient of the surfactant concentration at the interface (Fig. 9,a). Fig. 9,b 
corresponds to the beginning of the intensive vortex convection motion caused by an influx of the solution with higher alcohol content from the region over the drop. Note that the major mass of the alcohol entrained by the capillary convection is not absorbed by the drop. It remains in the surrounding solution and, being lighter than the adjacent layers, floats up. Separation of the alcohol flux from the drop surface occurs in the region where the action of the capillary forces is balanced by the Archimedean force, which increases as the surfactant moves downward. As in the case with a bubble, the rising solution forms two closed vortex cells. The alcohol penetrating the drop also forms an intensive two-cell flow (Fig. 9,c). Capturing an increasingly growing amount of solution with high surfactant concentration, the convective cells outside the drop become more and more light. As a result they move gradually upward until finally they cut off the feeding flux of the alcohol from the drop. This causes the Marangoni flow to slow down abruptly. Simultaneously, inside the drop, the solution of chlorobenzene with rather high alcohol content, held all this time at the lower part of the drop by a large-scale flow, begins to float up. If, by this time, the drop has been already saturated with the surfactant (i.e. within a few cycles after the beginning of the test) the buoying alcohol becomes excessive, and the drop ejects it back into the surrounding solution reaching thereby chemical equilibrium with the environment. Then, as in the case with a bubble, the gravitational force restores the vertical stratification of the surfactant near the drop surface and the cycle is repeated.

Time variation of the period of the flow intensification near the drop is presented in Fig. 10. As measurements show, an increase in the period with time is related to a decrease in the gradient of the surfactant concentration in the surrounding solution due to diffusive and convective mixing. Variation of the surfactant concentration gradient has a two-fold effect: it reduces the intensity of the capillary forces and increases the amount of the surfactant ejected by the drop at the end of the cycle. Fig. 11 presents time dependence of the ratio of the maximum alcohol concentration differences inside and outside the drop. As it is seen, this ratio remains practically constant for the whole period of observation ( $\sim 3$ hours) in spite of a very complicated flow behavior.

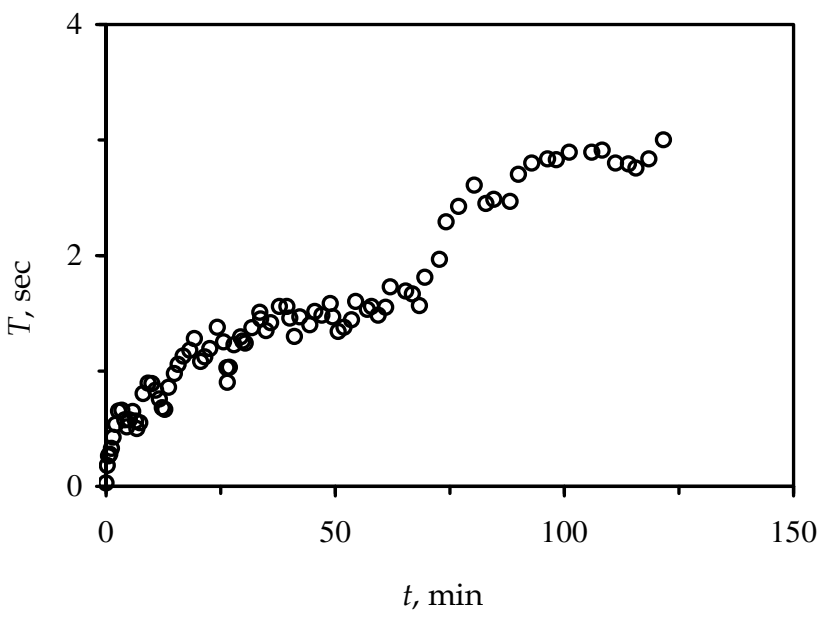

Fig. 10. The period of flow intensification near the drop as a function of time 


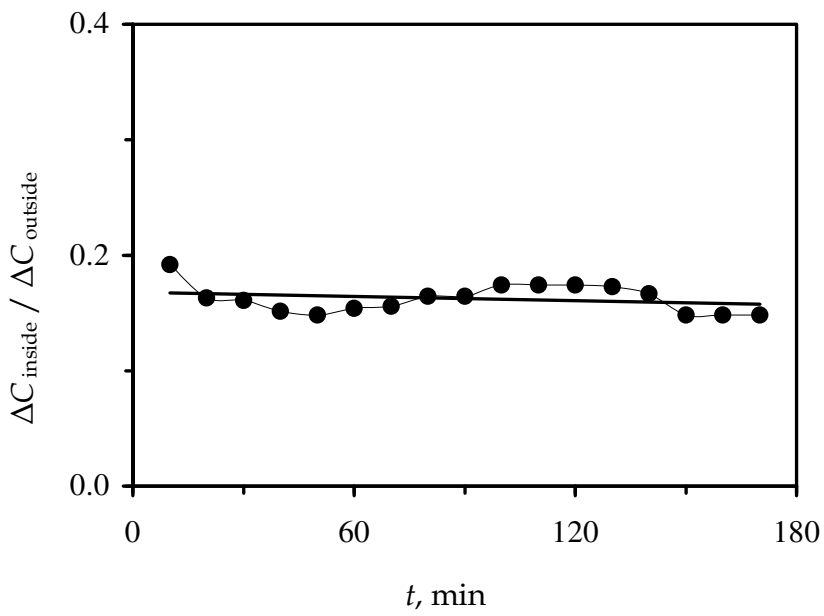

Fig. 11. Relationship between surfactant concentration inside and outside the drop

\section{Plane rectangular channel}

In our next experiments (Birikh et al., 2006, 2008; Bushueva et al., 2008; Kostarev et al., 2008a, 2009a) the fluid filled a thin gap between two vertically oriented glass plates. Inside the gap the fluid was bounded from above and below by solid plates, which formed a horizontal channel of height $h=2 \mathrm{~mm}$ and thickness $1.2 \mathrm{~mm}$. The tests were performed for aqueous solutions of methyl, ethyl and isopropyl alcohols. The channel was initially filled with distilled water or uniform alcohol solution with weight percent concentrations ranging from 1 to $10 \%$. An air bubble put into the channel cavity from one of its ends in such a way that it completely bridged over the channel and had one free lateral boundary. Then, from the other end, the cavity was gradually filled with a concentrated (up to $40 \%$ ) alcohol solution. The fluid density difference thus produced in the channel led to the formation of a rather slow large-scale advective gravitational flow, during which a narrow "tongue" of a more concentrated surfactant solution flowed along the upper channel boundary toward the bubble surface, forming near the surface an area with upward-directed concentration gradient. The value of the concentration gradient was specified by the velocity and concentration of the inflowing surfactant flux, as well as by the initial concentration of the solution in the channel.

The interference patterns of the concentration field near the lateral boundary of the air bubble is shown in Fig. 12 and Fig. 13. In contrast to thermocapillary flow developing in a non-threshold manner at arbitrary small values of the interface temperature gradient, the solutocapillary flow was initiated not at the time when the surfactant "tongue" reached the bubble surface, but with some delay. Thus, in the experiment a time-lag $\Delta t$ between the arrival of the surfactant flow at the bubble surface and the initiation of the convective vortex was as long as $28 \mathrm{sec}$. At the same time the difference of the solution concentration $\Delta C^{*}$ between the upper and lower bubble poles caused by a continued motion of the "tongue" reached $2.2 \%$. Then the equilibrium was abruptly disturbed and very rapidly (during about $0.2 \mathrm{sec}$ ) a rather intensive Marangoni flow was initiated in the form of a vortex cell, in which 
the surfactant under the action of solutocapillary forces was carried along the bubble surface toward the lower bubble boundary. Owing to fluid continuity, the arising flow accelerated the flux of the concentrated surfactant solution along the upper boundary of the channel towards the bubble surface, adding thereby intensity to the existing convective vortex. However, the originated vortex cell, entrapping more and more portions of highly concentrated surfactant solution, became increasingly light. Rising up it eventually cut off the arriving jet of surfactant from the top of the bubble. As a result, the vortex flow ceased abruptly and the bubble surface turned out to be surrounded by a thin layer of the surfactant solution having a uniform concentration.

a)

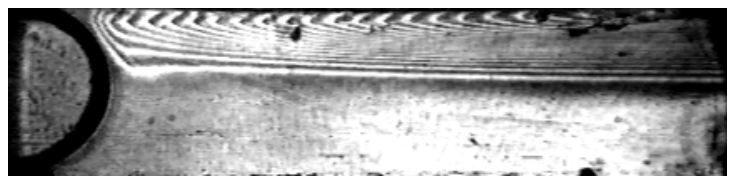

b)

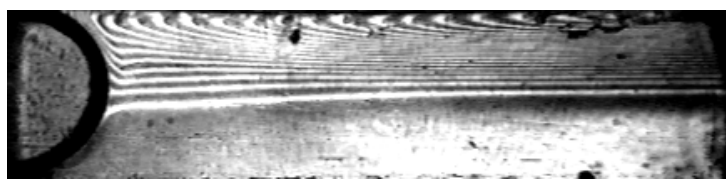

c)

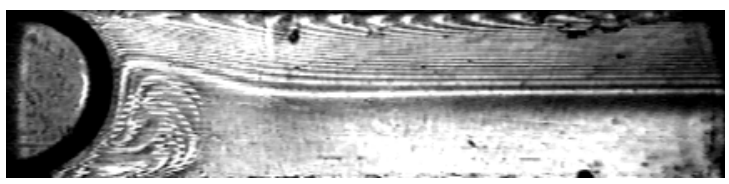

d)

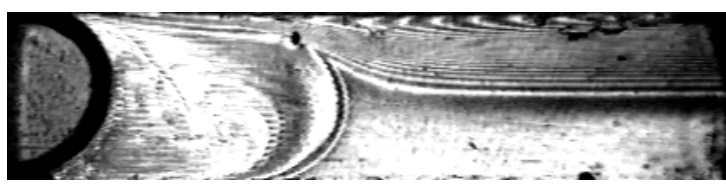

e)

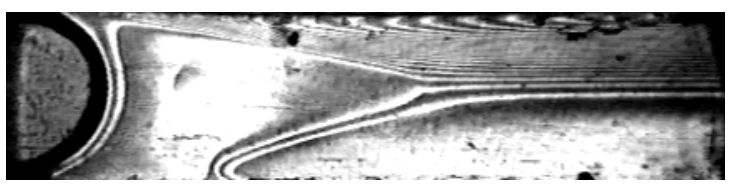

f)

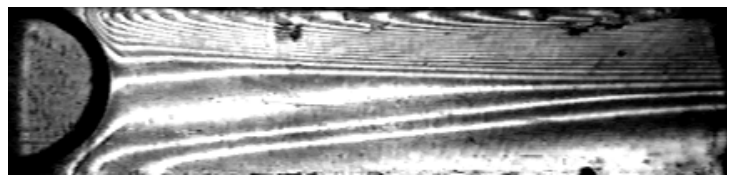

g)

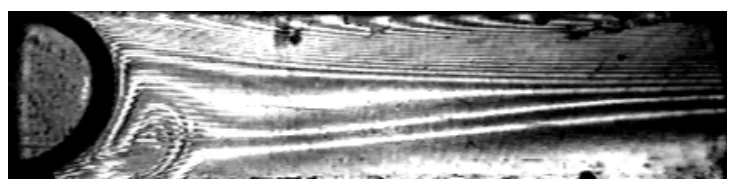

Fig. 12. Interferograms of concentration field evolution around the air bubble in rectangular layer with stratified isopropyl alcohol solution. $t$, sec: $0,28.0,28.1,28.2,29.2,34.2,34.3$ 
a)

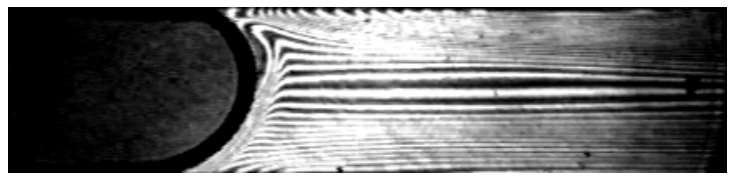

b)

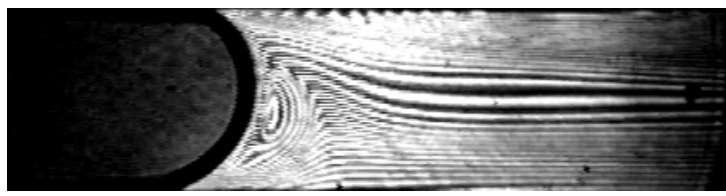

c)

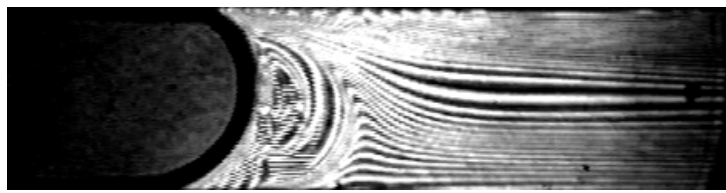

d)

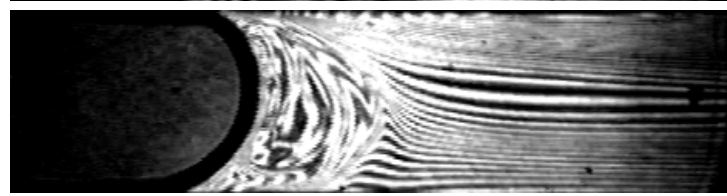

e)

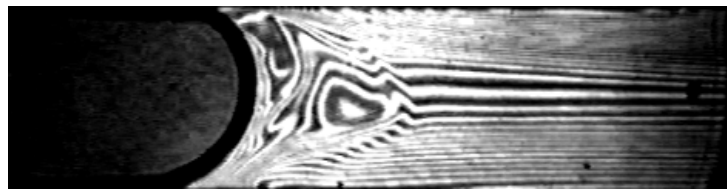

f)

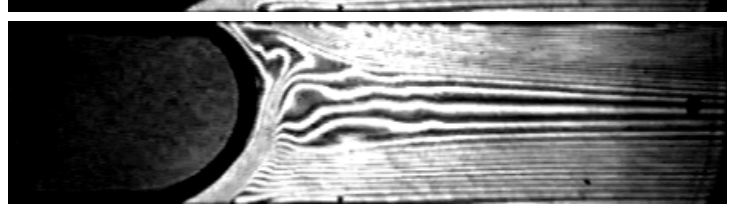

g)

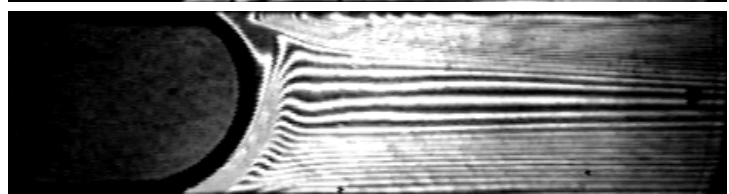

Fig. 13. Interferograms of concentration field evolution around the air bubble in rectangular layer with stratified ethyl alcohol solution. $t$, sec: $0,0.2,0.4,0.8,5.0,10.0,20.0$

However, equalizing of the generated horizontal gradient of the surfactant concentration resulted in the development of a slow advective flow. This gravitational motion, by restoring the disturbed vertical stratification of the solution, again draws a concentrated surfactant solution to the upper bubble boundary. As soon as the flux of surfactant touched the bubble surface, the solutocapillary vortex recurred. The cycle repeated iteratively, with the difference that the oscillation period increased with time whereas the intensity of the vortex flow decreased due to a gradual decrease of the vertical concentration gradient. Marangoni convection ceased at the time when the concentration of the solution became almost uniform throughout the whole layer. 
A characteristic feature of this process is that during consecutive cycles the capillary flow was initiated at much less concentration differences at the bubble surface (thus, the onset of the second cycle happened at $\Delta C^{*} \sim 0.6 \%$ ). At the same time, the average concentration of the surfactant at the bubble surface gradually increased, which might be one of the explanations for the essential decrease in the concentration difference at the bubble surface at the beginning of each consecutive cycle of the vortex convection motion. In order to verify this suggestion we investigated the dependence of the critical Marangoni numbers $M a^{*}$, defined by the maximum vertical concentration difference between the bubble poles at the moment of formation of the first vortex, on the surfactant concentration in the solution surrounding the bubble. To this purpose, the channel was initially filled not with pure water but with a homogeneous aqueous solutions of alcohols with different initial concentration $C_{0}$. It was found that, as $C_{0}$ increases (and, respectively, as the surface tension at the bubble surface decreases) the values of $M a^{*}$ decrease monotonically. Fig. 14 presents the critical Marangoni numbers at the beginning of different cycles of the vortex flow in solutions of ethanol and isopropanol (points 1 and 2) as functions of the crispation number $\mathrm{Cr}$, characterizing the ratio of viscous and capillary forces. The crispation numbers, in turn, were calculated using the values of the surface tension corresponding to the average surfactant concentration at the air bubble surfaces at the moments of motion intensification. The diagram also presents the results of measurements of $M a^{*}$ as a function of $\mathrm{Cr}$, obtained from tests for solutions with different initial alcohol concentration (solid line drawn through points 3 for ethanol and 4 for isopropanol). It is seen that the values of $M a^{*}$ obtained under various conditions are rather close and all curves are qualitatively coincide.

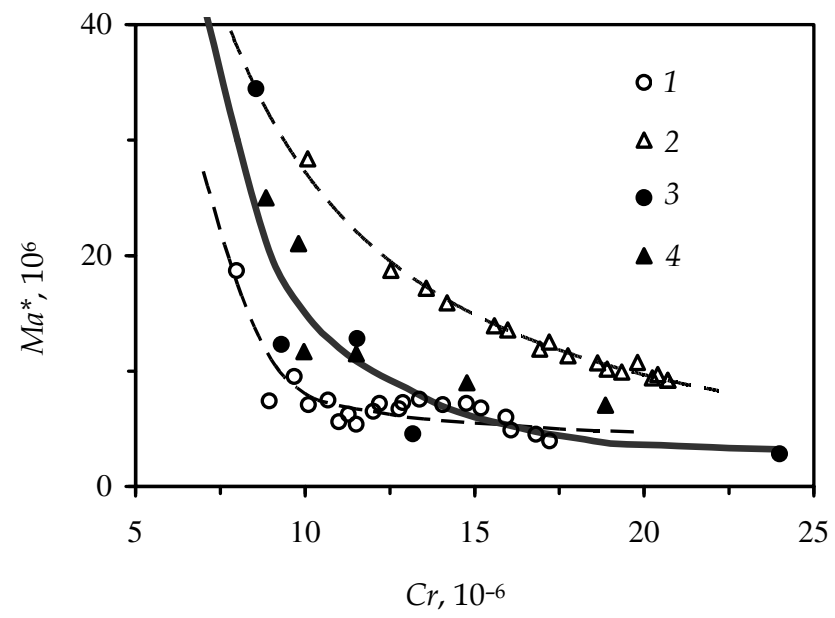

Fig. 14. Critical Marangoni numbers as a function of crispation number

In our experiments we also investigated the time dependence of the oscillation period of the convective flow near the bubble in solutions of ethanol, isopropanol and methanol. In all cases, the oscillation frequency of the flow near the bubble surface was first rather high (accordingly the period $T$ was small $\sim 5-10 \mathrm{sec}$ ). Then, the oscillation period increased 
monotonically with time, and after some time the oscillations suddenly came to an end. The experimental data obtained were compared with the results of numerical analysis (Birikh et al., 2006). The results of experiments and numerical calculations were found to be in good agreement with respect to the convective flow structure and the oscillation period. The period of the steady-state oscillations decreases with an increase of the Grashof number and depends weakly on the Marangoni number. This dimensionless relationship is represented in Fig. 15. The different points on the plot represent the results of experiments for various alcohol solutions ( 1 - ethanol at $\mathrm{C}_{0}=40 \%, 2$ - ethanol at $\mathrm{C}_{0}=20 \%, 3$ - isopropanol, 4 methanol). The solid line corresponds to numerical calculations.

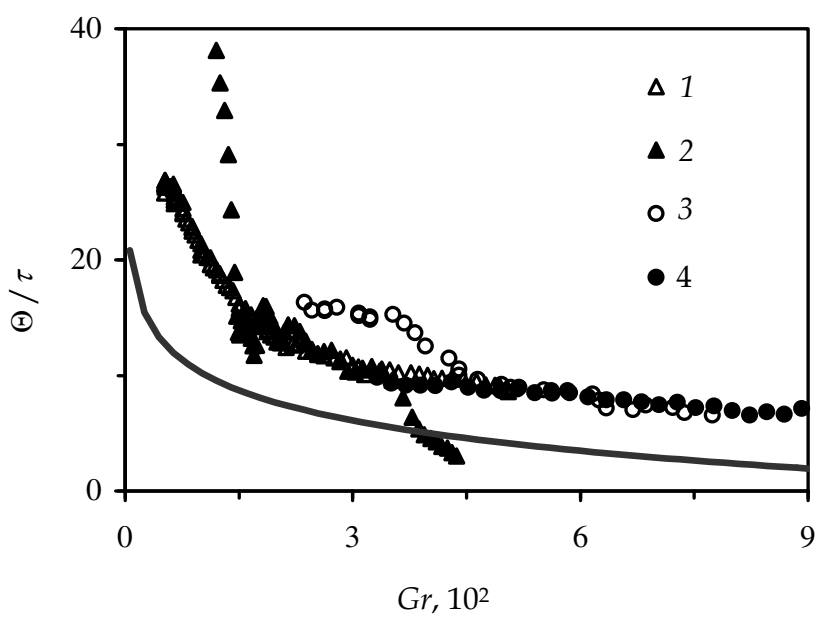

Fig. 15. Dimensionless oscillation period

An analogous situation was observed on the chlorobenzene - aqueous isopropanol solution interface (Kostarev et al., 2008b). A chlorobenzene drop, immiscible in the water, was injected by means of a syringe into a channel cavity from one of its ends in such a way that completely bridged over the channel and formed lateral liquid/liquid boundary. A "tongue" of a concentrated (40\%) isopropyl solution flowed along the upper channel boundary toward the drop surface, forming near it an area of nixture with vertical surfactant stratification. In contrast with the convection initiation around the gas bubble, a much more greater surfactant concentration gradient is needed to be created during the time between the moments when the alcohol reaches the droplet surface and before solutocapillary motion begins to develop, as is seen in the interferograms in Fig. 16. The difference of the solution concentration $\Delta C^{*}$ between the upper and lower drop ends equalized to $4.1 \%$ against the value of $2.2 \%$ in case of gas bubble. The other distinction is that surfactant diffuses through the phase interface into chlorobenzene, thus creating a concentration gradient in the latter also. Initially, this process occurs rather uniformly along the droplet surface, so that a surfactant gradient at the surface itself is absent. Only later, an intense Marangoni convection develops in the solution (at $\Delta t \sim 1 \mathrm{~min}$ after the contact between the surfactant tongue and the droplet surface. 
a)

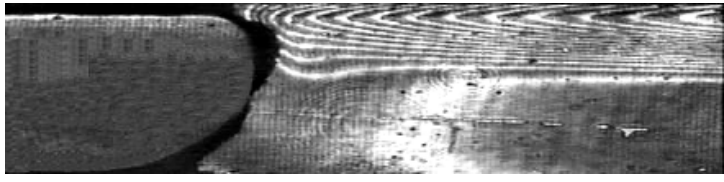

b)

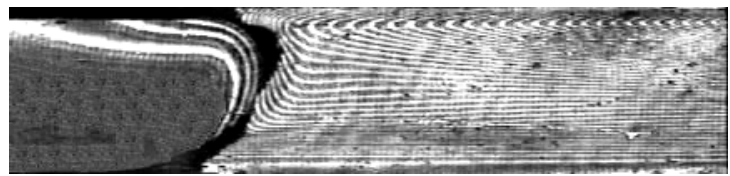

c)

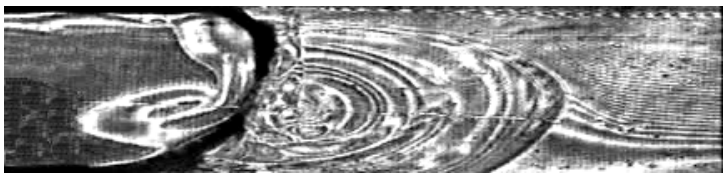

d)

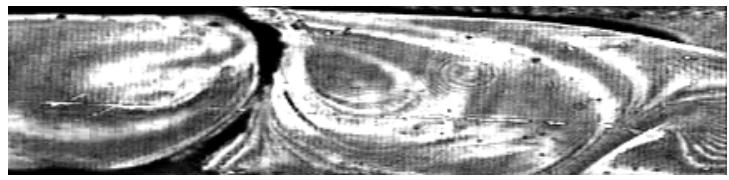

e)

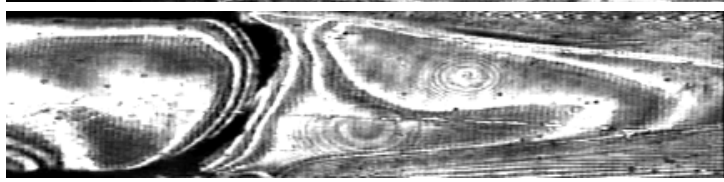

f)

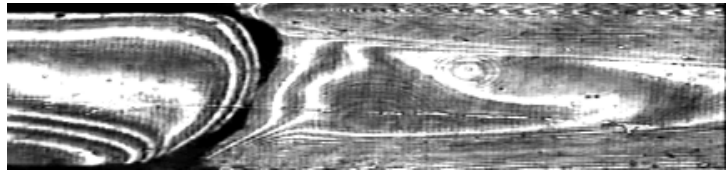

g)

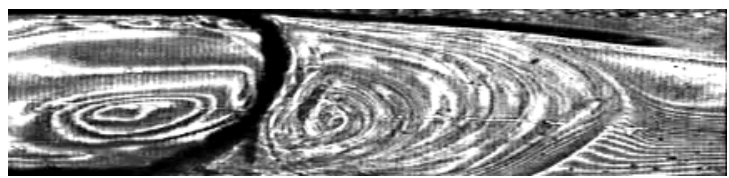

Fig. 16. Interferograms of the concentration field in the experiment with a chlorobenzene droplet in a stratified aqueous isopropanol solution: $t$, sec: $0,60,61,66,70,76,77$

\section{Conclusion}

The performed experiments provided convincing evidence for the origination of intensive solutocapillary Marangoni convection in multiphase systems with inhomogeneous concentration of the soluble surfactant along the phase interface. The specific feature of these fluid flows was that they took place under conditions of weak surfactant diffusion (the values of the Schmidt number in experiments were about $10^{3}$ ), and that the changes in the spatial concentration of the solution happened mainly due to convective mass transfer. The arising stresses and flows in some ways are similar to thermocapillary ones and are governed by similar relationships, for example, flow intensity is proportional to the surface tension gradient. On the other hand, the solutocapillary phenomena demonstrate some specific features, which are related both to fairly large values of the Marangoni numbers and 
to a more complicated character of formation of the surface tension gradient. The mechanism of the transfer (absorption) of a surfactant at the phase interface is distinct from the mechanism maintaining the temperature at the interface. The interface has inertia, and a convective transfer of the surfactant is possible along it, accompanied by surface diffusion. The latter peculiarity is caused by slower (compared to heat transfer) diffusion of the fluid molecules with low surface tension onto the interface and adsorption of the surfactant at the inter-phase boundary. These specific features should be taken into account in constructing theoretical models of the boundary conditions at the interface allowing for the formation of a new, surface phase of the surfactant, controlling a transition of the soluble mixture component from one phase to another. The process of formation of such a surface phase includes two stages. First, the molecules of the surfactant reach the interface of the liquid system via diffusive transport, because the normal component of the convective velocity at the surface is zero due to impermeability of the boundary. This process is followed then by formation of the proper interface in itself, by means of adsorption and desorption.

The experiments demonstrated that the solutocapillary convection displays well-defined non-stationary properties. The interaction between the buoyancy and the Marangoni convective flows is responsible for the onset of auto-oscillation regime of convective motion around the gas bubbles and insoluble drops in a vertically stratified surfactant solutions. The periodical outbursts of solutocapillary flow at the bubbles/drops interface intensify substantially the stirring of the solution and can be used for the control of this procedure. The clarification of the nature of the flows developing close to resting liquid and gaseous inclusions in inhomogeneous surfactant solutions and the discovery of a threshold for the excitation of solutocapillary motion allow explaining many aspects of mass exchange and material structure formation in a number of technological experiments in microgravity, and predicting the behavior of complex systems of liquids and multiphase media in thin channels and layers, and in cavities of complex geometry. The results obtained in the experiments can be used in designing systems of passive homogenization in liquids and in optimizing working regimes of the already operational technology lines in various branches of industry. A special role can be played by the Marangoni effects elaborated here in the design of microsystems for cooling and heat exchange with multi-component mixtures of liquids as a heat agent.

\section{Acknowledgements}

The work was supported by Russian Foundation of Fundamental Research under project No 09-01-00484, joint project of SB, UB and F-EB of RAS (116/09-C-1-1005) and the program of the Department of Power Engineering, Mechanical Engineering, Mechanics and Control Processes of RAS No 09-T-1-1005.

\section{References}

Birikh, R.V.; Zuev, A.L.; Kostarev, K.G. \& Rudakov, R.N. (2006). Convective self- oscillations near an air-bubble surface in a horizontal rectangular channel. Fluid Dynamics, Vol. 41, No. 4, pp. 514-520.

Birikh, R.V.; Rudakov, R.N.; Kostarev, K.G. \& Zuev, A.L. (2008). Oscillatory modes of solutocapillary Marangoni convection at a drop-liquid interface. Proceedings of $6^{\text {th }}$ 
EUROMECH Nonlinear Dynamics Conf., pp. 1-6., Saint Petersburg, Russia, 30 June 4 July 2008.

Bushueva, K.A.; Denisova, M.O.; Zuev, A.L. \& Kostarev, K.G. (2008). Flow development at the surfaces of bubbles and droplets in gradient solutions of liquid surfactants. Colloid J., Vol. 70, No. 4., pp. 416-422.

Gustafson, S.E.; Kjellander R.A.E. \& Rolf A.E. (1968). An interferometer for direct recording of refractive index distributions. Z. Naturforch., Vol. 23a, No. 2, pp. 242-246.

Kostarev, K.G.; Zuev, A.L. \& Viviani, A. (2004). Oscillatory Marangoni convection around the air bubble in a vertical surfactant stratification. Comptes Rendus Mecanique, Vol. 332, No. 1, pp. 1-7.

Kostarev, K.G.; Zuev, A.L. \& Viviani, A. (2006). Thermal and concentrational Marangoni convection at liquid/air bubble interface. J. Applied Mechanics. Transactions ASME, Vol. 73, No. 1, pp. 66-71.

Kostarev, K.G., Pisarevskaya, N. N., Viviani, A. \& Zuev, A. L. (2007). Oscillatory Marangoni convection around bubbles and drops in heterogeneous solutions of surfactants. Int. J. Microgravity Science \& Technology, Vol. 19, No. 2, pp. 12-17.

Kostarev, K.G.; Zuev, A.L. \& Viviani, A. (2008a). Experimental study of convective selfoscillations near the lateral surface of a bubble in a plane rectangular channel. Acta Astronautica, Vol. 62, No. 6-7, pp. 431-437.

Kostarev, K.G.; Zuev, A.L. \& Viviani, A. (2008b) Experimental studies of concentration convective surfactant mass transfer near a drop-liquid interface. Proceedings of $19^{\text {th }}$ Int. Symp. on Transport Phenomena, pp. 1-5, Reykjavik, Iceland, 17-21 August 2008. (In Electronic Conference Proceedings).

Kostarev, K.G.; Zuev, A.L. \& Viviani, A. (2009a). Experimental considerations of solutocapillary flow initiation on bubble/drop interface in the presence of a soluble surfactant. Int. J. Microgravity Science and Technology, Vol. 21, No. 1-2, pp. 59-65.

Kostarev, K.G.; Zuev A.L. \& Viviani A. (2009b). Convective stirring of a stratified surfactant solution by the oscillatory solutocapillary flow. Proceedings of $4^{\text {th }}$ Int. Conf. on Physics and Control, pp. 1-8, Catania, Italy, 1-4 September 2009. (In Online Conference Proceedings http://lib.physcon.ru/download / p1930.pdf).

Marsters, G.F. \& Advani, A.A. (1973). A tilted plate interferometer for heat transfer studies. Rev. Sci. Instrum., Vol. 44, No. 8, pp. 1015-1018.

Vazquez, G.; Alvarez, E. \& Navaza, J.M. (1995). Surface-tension of alcohol plus water from 20-degrees-C to 50-degrees-C. J. Chem. Eng. Data, Vol. 40, No. 3, pp. 611-614.

Vazquez, G.; Alvarez, E.; Sanchez-Vilas. M.; Sanjurjo B. \& Navaza J.M. (1997). Surface tension of organic acids + water binary mixtures from $20^{\circ} \mathrm{C}$ to $50^{\circ} \mathrm{C}$. J. Chem. Eng. Data, Vol. 42, No. 5, pp. 957-960.

Zuev, A.L. \& Kostarev, K.G. (2006). Oscillation of a convective flow round the air bubble in a vertically stratified solution of a surfactant. J. Experimental and Theoretical Physics, Vol. 130, No. 2, pp. 363-370.

Zuev, A. L.; Kostarev, K.G. \& Viviani, A. (2008a). Peculiarities of the solutocapillary convection. Proceedings of $11^{\text {th }}$ Int. Conf. on Multiphase Flow in Industrial Plants, pp. 39-46, Palermo, Italy, 7-10 September 2008

Zuev, A.L. \& Kostarev, K.G. (2008b). Certain peculiarities of the solutocapillary convection, Physics-Uspekhi, Vol. 51, No. 10, pp. 1027-1045. 


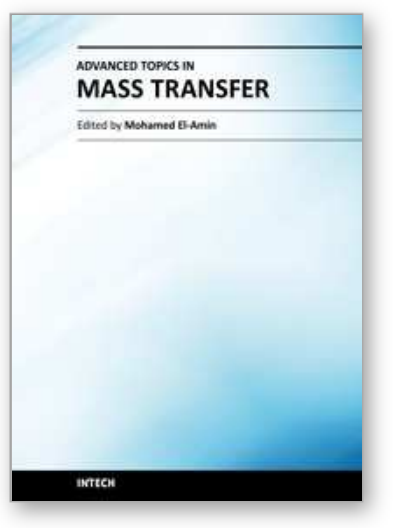

\author{
Advanced Topics in Mass Transfer \\ Edited by Prof. Mohamed El-Amin
}

ISBN 978-953-307-333-0

Hard cover, 626 pages

Publisher InTech

Published online 21, February, 2011

Published in print edition February, 2011

This book introduces a number of selected advanced topics in mass transfer phenomenon and covers its theoretical, numerical, modeling and experimental aspects. The 26 chapters of this book are divided into five parts. The first is devoted to the study of some problems of mass transfer in microchannels, turbulence, waves and plasma, while chapters regarding mass transfer with hydro-, magnetohydro- and electro- dynamics are collected in the second part. The third part deals with mass transfer in food, such as rice, cheese, fruits and vegetables, and the fourth focuses on mass transfer in some large-scale applications such as geomorphologic studies. The last part introduces several issues of combined heat and mass transfer phenomena. The book can be considered as a rich reference for researchers and engineers working in the field of mass transfer and its related topics.

\title{
How to reference
}

In order to correctly reference this scholarly work, feel free to copy and paste the following:

Konstantin Kostarev, Andrew Zuev and Antonio Viviani (2011). Oscillatory Regimes of Solutocapillary Marangoni Convection, Advanced Topics in Mass Transfer, Prof. Mohamed El-Amin (Ed.), ISBN: 978-953-307333-0, InTech, Available from: http://www.intechopen.com/books/advanced-topics-in-mass-transfer/oscillatoryregimes-of-solutocapillary-marangoni-convection

\section{INTECH}

open science | open minds

\author{
InTech Europe \\ University Campus STeP Ri \\ Slavka Krautzeka 83/A \\ 51000 Rijeka, Croatia \\ Phone: +385 (51) 770447 \\ Fax: +385 (51) 686166 \\ www.intechopen.com
}

\author{
InTech China \\ Unit 405, Office Block, Hotel Equatorial Shanghai \\ No.65, Yan An Road (West), Shanghai, 200040, China \\ 中国上海市延安西路65号上海国际贵都大饭店办公楼405单元 \\ Phone: +86-21-62489820 \\ Fax: +86-21-62489821
}


(C) 2011 The Author(s). Licensee IntechOpen. This chapter is distributed under the terms of the Creative Commons Attribution-NonCommercialShareAlike-3.0 License, which permits use, distribution and reproduction for non-commercial purposes, provided the original is properly cited and derivative works building on this content are distributed under the same license. 